\title{
COMPLETE CONVERGENCE FOR WEIGHTED SUMS OF ARRAYS OF RANDOM ELEMENTS
}

\author{
ROBERT LEE TAYLOR \\ Department of Mathematics and Statistics \\ University of South Carolina \\ Columbia, S. C. 29208 \\ (Received Ju1y 22, 1982)
}

ABSTRACT. Let $\left\{\mathrm{x}_{\mathrm{nk}}: \mathrm{k}, \mathrm{n}=1,2, \ldots\right\}$ be an array of row-wise independent random elements in a separable Banach space. Let $\left\{a_{n k}: k, n=1,2, \ldots\right\}$ be an array of real numbers such that $\sum_{\mathrm{k}=1}^{\infty}\left|\mathrm{a}_{\mathrm{nk}}\right| \leq 1$ and $\Gamma_{L_{\mathrm{n}=1}}^{\infty} \exp \left(-\alpha / \mathrm{A}_{\mathrm{n}}\right)<\infty$ for each $\alpha \varepsilon \mathrm{R}^{+}$where $A_{n}=\Gamma_{k=1}^{\infty} a_{n k}^{2}$. The complete convergence of $l_{l_{k=1}}^{\infty} a_{n k} x_{n k}$ is obtained under varying moment and distribution conditions on the random elements. In particular, laws of large numbers follow for triangular arrays of random elements, and consistency of the kernel density estimates is obtained from these results.

KEY WORDS AND PHRASES. Weighted Sums, Random Elements, Laws of Large iumbers, subGaussian, Complete Convergence, and Kemel Density Estimates.

1980 MATHEMATICS SUBJECT CLASSIFICATION CODES. Primary 60B12, Secondary $60 B 11$.

\section{INTRODUCTION AND PRELIMINARIES.}

Wei and Taylor [1] obtained the convergence of $\sum_{k=1}^{\infty} a_{n k} x_{k}$ in probability and with probability one by assuming tightness (given $\varepsilon>0$ there exists a compact set $\mathrm{K}_{\varepsilon}$ such that $\sup _{n} \mathrm{P}\left[\mathrm{X}_{\mathrm{n}} \notin \mathrm{K}_{\varepsilon}\right]<\varepsilon$ and uniformly bounded $\mathrm{p}^{\text {th }}(\mathrm{p}>1)$ moment conditions on the sequence of random elements $\left\{\mathrm{x}_{\mathrm{n}}\right\}$ in separable Banach spaces. Howell and Taylor [2] proved Marcinkiewicz-Zygmund type weak laws of large numbers for the weighted sum $\sum_{\mathrm{k}=1}^{\mathrm{n}} \mathrm{a}_{\mathrm{nk}} \mathrm{x}_{\mathrm{nk}}$ of arrays of random elements $\left\{\mathrm{x}_{\mathrm{nk}}\right\}$ in Banach spaces satisfying certain geometric conditions. In this paper a stronger mode of convergence, complete convergence, is obtained for $\sum_{k=1}^{\infty} a_{n k} x_{n k}$ in separable Banach spaces with less restrictive conditions than the results of Wei and Taylor and without assuming geometric 
conditions on the spaces. The format for these results was motivated in part by the form of the kernel density estimates (which are weighted sums of arrays of random elements) and by the relative absence of laws of large numbers for arrays of random elements.

Let $\mathrm{E}$ denote a real separable Banach space with norm \|\| . Let $(\Omega, A, P)$ denote a probability space. A random element $X$ in $E$ is a function from $\Omega$ into $E$ which is A-measurable with respect to the Borel subsets of $E$. The expected value of $X$ is defined to be the Pettis integral (when it exists, see pp. 38-41 of [3]) and is denoted by EX. The $\mathrm{p}^{\text {th }}$ moment of a random element $\mathrm{X}$ is $\mathrm{E}\left(\left.\|\mathrm{X}\|\right|^{\mathrm{P}}\right)$ where $\mathrm{E}$ is the expected value of the (real-valued) random variable $\|x\|^{P}$. The concepts of independence and identical distributions (i.i.d.) have direct extensions to E. A sequence of random elements $\left\{\mathrm{X}_{\mathrm{n}}\right\}$ is said to converge completely to the random element $\mathrm{X}$ if

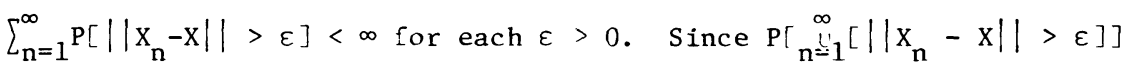
$\leq \sum_{n=1}^{\infty} P\left[|| X_{n}-X||>\varepsilon\right]<\infty$, complete convergence implies convergence with probability one, but the reverse implication need not hold. Because of the methods of proof, complete convergence will be obtained for the results of this paper rather than (the standard) convergence with probability one.

\section{The Complete Convergence of Weighted Sums.}

In this section complete convergence for weighted sums of arrays of random elements is obtained. Since no geometric conditions are assumed on the space, it is easy to show that moments conditions alone will not suffice. Thus, sigma compact support or uniform compact integral conditions will be assumed on the distributions of the random elements. Throughout this section $\left\{\mathrm{x}_{\mathrm{nk}}\right\}$ will denote an array of random elements in a separable Banach space $E$ such that $\left\{x_{n k}: k \geq 1\right\}$ are independent for each $n$. Moreover, $\left\{a_{n k}\right\}$ will always denote an array of real numbers such that

$$
\sum_{k=1}^{\infty}\left|a_{n k}\right| \leq 1 \text { and } \sum_{n=1}^{\infty} \exp \left[-\alpha / A_{n}\right]<\infty
$$

for each $\alpha>0$ where $A_{n}=\sum_{\cdot k=1}^{\infty} a_{n k}^{2}$.

First, the complete convergence of the weighted sums will be obtained when the random elements are restricted to a compact subset with probability one. This result will allow consideration of the random elements which are truncated to compact subsets 
and the corresponding parts off the compact subsets.

THOREM 1. Let $\mathrm{K}$ be a compact subset of a separable Banach space $\mathrm{E}$. If $\left\{\mathrm{X}_{\mathrm{nk}}\right\}$ is an array of random elements in $E$ such that $\left\{x_{n k}: k \geq 1\right\}$ are independent for each $\mathrm{n}$ and such that $\mathrm{P}\left[\mathrm{X}_{\mathrm{nk}} \in \mathrm{K}\right]=1$ and $\mathrm{EX}_{\mathrm{nk}}=0$ for all $\mathrm{n}$ and $\mathrm{k}$, then,

$$
\left\|\sum_{k=1}^{\infty} a_{n k} x_{n k}\right\| \rightarrow 0 \text { completely. }
$$

PROOF. It can be assumed that $\mathrm{K}$ is convex and symmetric and $0 \in \mathrm{K}$ (wlog). In the dual space $E^{*}$, there is a countable set $S=\left\{f_{i}\right\}$, with $\left\|f_{i}\right\|=1$, which separate points of $K$. Also, for each $\varepsilon>0$ there exists $\left\{f_{1}, \ldots, f_{m}\right\} \subset s$ such that

$$
\{\mathrm{x} \in \mathrm{K}:\|\mathrm{x}\|>\varepsilon\} \subset \mathrm{i}_{\mathrm{i}=1}^{\mathrm{m}}\left\{\mathrm{x} \in \mathrm{K}:\left|\mathrm{f}_{\mathrm{i}}(\mathrm{x})\right|>\frac{\varepsilon}{2}\right\} .
$$

Next, for each $n \sum_{k=1}^{\infty}\left|a_{n k}\right| \leq 1$ implies by convexity that $\sum_{k=1}^{\infty} a_{n k} x_{n k} \epsilon \mathrm{K}$ with probability one. Hence, for each $\varepsilon>0$ and each $n$

$$
\begin{aligned}
P\left[|| \sum_{k=1}^{\infty}\right. & \left.a_{n k} x_{n k} \|>\varepsilon\right] \\
& \leq \sum_{i=1}^{m} P\left[\left|\sum_{k=1}^{\infty} a_{n k} f_{i}\left(x_{n k}\right)\right|>\frac{\varepsilon}{2}\right] .
\end{aligned}
$$

For each $f_{i}, E\left[f_{i}\left(x_{n k}\right)\right]=0$ for all $n$ and $k$, and $\left|f_{i}\left(x_{n k}\right)\right| \leq\left\|f_{i}\right\|\left(\sup _{x \in K}\|x\|\right)=$ with probability one. Hence,

$$
E\left[\exp \left(t f_{i}\left(x_{n k}\right)\right)\right] \leq e^{2} t^{2},
$$

or $\left\{\mathrm{f}_{i}\left(\mathrm{x}_{\mathrm{nk}}\right) / \Gamma\right\}$ are sub-Gaussian with parameters $\leq 2^{1 / 2}$ for each $i$. By Theorem 2 of Chow [4],

$$
\frac{1}{\Gamma} \sum_{k=1}^{\infty} a_{n k} f_{i}\left(x_{n k}\right) \rightarrow 0 \quad \text { completely }
$$

for each $f_{i}$. Combining (2.3) and (2.5), it follows that for each $\varepsilon>0$

$$
\sum_{n=1}^{\infty} P\left[|| \sum_{k=1}^{\infty} a_{n k} x_{n k} \|>\varepsilon\right]<\infty
$$

Using truncation to a compact subset and Theorem 1, a strong law of large numbers will be proved for triangular arrays of random elements.

THEOREM 2. Let $\left\{\mathrm{x}_{\mathrm{nk}}\right\}$ be an array of random elements in a separable Banach space E satisfying 
(i) $\left\{\mathrm{x}_{\mathrm{nk}}: \mathrm{k} \geq 1\right\}$ are i.i.d. for each $\mathrm{n}$,

(ii) $\mathrm{EX}_{\mathrm{nk}}=0$ for all $\mathrm{n}$ and $\mathrm{k}$,

(iii) $\sum_{n=1}^{\infty} E\left(\left\|x_{n 1}\right\|^{2 q}\right) / n^{q}<\infty$ for some $q \geq 1$, and

(iv) given $\varepsilon>0$ there exists a compact subset $\mathrm{K}_{\varepsilon}$ such that

Then,

$$
\lim _{\mathrm{n}} \sup E\left(\left\|\mathrm{X}_{\mathrm{n} 1}\right\| \mathrm{I}_{\left[\mathrm{X}_{\mathrm{n} 1} \notin \mathrm{K}\right]}\right)<\varepsilon \text {. }
$$

$$
\left\|\frac{1}{n} \sum_{k=1}^{n} x_{n k}\right\| \rightarrow 0 \quad \text { completely. }
$$

PROOF. Given $\varepsilon>0$ choose $K_{\varepsilon}$ compact, convex, and symmetric with $0 \in \mathrm{K}_{\varepsilon}$ such that

$$
\left.\underset{\mathrm{n}}{\lim \sup } \frac{1}{\mathrm{n}} \sum_{\mathrm{k}=1}^{\mathrm{n}} \mathrm{E}\left(\left\|\mathrm{x}_{\mathrm{nk}}\right\| \mathrm{I}_{\left[\mathrm{x}_{\mathrm{nk}}\right.} \notin \mathrm{K}_{\varepsilon}\right]\right)<\frac{\varepsilon}{8}
$$

by (iv) and (i). Define

$$
\mathrm{Y}_{\mathrm{nk}}=\mathrm{X}_{\mathrm{nk}} \mathrm{I}_{\left[\mathrm{x}_{\mathrm{nk}} \in \mathrm{K}_{\varepsilon}\right]} \text { and } \mathrm{Z}_{\mathrm{nk}}=\mathrm{x}_{\mathrm{nk}} \mathrm{I}_{\left[\mathrm{x}_{\mathrm{nk}} \notin \mathrm{K}_{\varepsilon}\right]^{\cdot}}
$$

Using $a_{n k}=\frac{1}{n}$ for $k=1, \ldots, n$ and $a_{n k}=0$ for $k>n$, it follows that $A_{n}=\frac{1}{n}$ and

$$
\sum_{n=1}^{\infty} \exp \left[-\alpha j A_{n}\right]=\sum_{n=1}^{\infty} \exp \left[-\alpha_{n}\right]<\infty .
$$

Since $\left\{\mathrm{Y}_{\mathrm{nk}}-\mathrm{EY}_{\mathrm{nk}}\right\}$ take their values in $2 \mathrm{~K}$,

$$
\sum_{n=1}^{\infty} P\left[|| \sum_{k=1}^{\infty} a_{n k}\left(Y_{n k}-E Y_{n k}\right) \|>\frac{\varepsilon}{2}\right]<\infty
$$

by Theorem 1 .

For each $\mathrm{n}$ and $\mathrm{k}$

$$
E\left[\left|\left\|z_{n k}\right\|-E\left\|z_{n k}\right\|\right|^{2 q}\right] \leq 2^{2 q} E\left\|x_{n k}\right\|^{2 q} \text {. }
$$

Thus, for each $\varepsilon>0$ and each $\mathrm{n}$

$$
\begin{aligned}
P\left[\left|\frac{1}{n} \sum_{k=1}^{n}\left(|| z_{n k}||-E|| z_{n k}||\right)\right|>\frac{\varepsilon}{4}\right] \\
\quad \leq(\varepsilon n / 4)^{-2 q} E\left[\left.||_{k=1}^{n}\left(|| z_{n k}\left\|-E|| z_{n k}\right\|\right)\right|^{2 q}\right] \\
\quad \leq(\varepsilon n / 4)^{-2 q} c_{1} n^{q} E\left[||\left|z_{n 1}\left\|-E|| z_{n 1}\right\|\right|^{2 q}\right]
\end{aligned}
$$

by Marcinkiewicz-Zygmund's inequality ( $r f$ : Corollary 2.1, Woyczynski [5]). 
From (2.9), (2.10) and (iii),

$$
\begin{gathered}
\sum_{n=1}^{\infty} P\left[\left|\frac{1}{n} \sum_{k=1}^{n}\left(|| z_{n k}\left\|-E|| z_{n k}\right\|\right)\right|>\frac{\varepsilon}{4}\right] \\
\leq C_{2} \sum_{n=1}^{\infty} E\left(\left\|x_{n 1}\right\|^{2 q}\right) / n^{q}<\infty
\end{gathered}
$$

where $c_{2}$ is a constant independent of $n$. Finally,

$$
\begin{aligned}
\sum_{n=1}^{\infty} & P\left[\left\|\frac{1}{n} \sum_{n=1}^{n} x_{n k}\right\|>\varepsilon\right] \\
& \leq \sum_{n=1}^{\infty} P\left[\left\|\frac{1}{n} \sum_{k=1}^{n}\left(Y_{n k}-E Y_{n k}\right)\right\|>\frac{\varepsilon}{2}\right] \\
& +\sum_{n=1}^{\infty} P\left[\left|\frac{1}{n} \sum_{k=1}^{n}\left(|| z_{n k}\left\|-E|| z_{n k}\right\|\right)\right|>\frac{\varepsilon}{4}\right] \\
& +\sum_{n=1}^{\infty} P\left[2 \frac{1}{n} \sum_{k=1}^{n} E\left\|z_{n k}\right\|>\frac{\varepsilon}{4}\right] \\
& <\infty
\end{aligned}
$$

from (2.6), (2.8), (2.11) and the fact that $\left\|E_{n k}\right\|=\left\|-E Z_{n k}\right\| \leq E\left\|z_{n k}\right\|$ for all $\mathrm{n}$ and $\mathrm{k}$.

The form of Theorem 2 is very appropriate for applications in kernel density estimation where $\left\{x_{1}, \ldots, x_{n}\right\}$ is a random sample from a distribution with unknown probability density function $f$. The kernel estimate for $f$ is given by

$$
\hat{f}_{n}(t)=\frac{1}{n h_{n}} \sum_{i=1}^{n} K\left(\frac{t-X_{i}}{h_{n}}\right)
$$

where $\mathrm{K}$ is an arbitrary kernel function which is usually assumed to be bounded, nonnegative and integrable to 1 and where $h_{n}$ is a sequence of positive numbers tending to zero which adjusts the height and spread of the kernel function. The random elements $\left\{\mathrm{x}_{\mathrm{nk}}\right\}$ in an appropriate Banach subspace of function space can be defined as $x_{n k}=\frac{1}{h_{n}} K\left(\frac{t-x_{k}}{h_{n}}\right)-\frac{1}{h_{n}} E K\left(\frac{t-x_{k}}{h_{n}}\right)$. Conditions (i) and (ii) of Theorem 2 are trivially satisfied. For complete convergence in the $L^{1}$-norm, (iii) is easily satisfied since

$$
E|| x_{n 1} \|^{2 q}=E\left(\int_{-\infty}^{\infty}\left|\frac{1}{h_{n}}\left(K\left(\frac{t-x_{1}}{h_{n}}\right)-E K\left(\frac{t-x_{1}}{h_{n}}\right)\right)\right| d t\right)^{2 q}
$$




$$
\begin{aligned}
& \leq E\left(\int_{-\infty}^{\infty} K\left(s-X_{1} / h_{n}\right) d s+\int_{-\infty}^{\infty} E K\left(\frac{t-X_{1}}{h_{n}}\right) \frac{1}{h_{n}} d t\right)^{2 q} \\
& =E(1+1)^{2 q}=2^{2 q} .
\end{aligned}
$$

In the sup-norm, $E|| x_{n 1}||^{2 q} \leq\left[\frac{2}{h_{n}} \text { bound }(K)\right]^{2 q}$, and thus $\sum_{n=1}^{\infty}\left(n_{n}{ }^{2}\right)^{-q}<\infty$ for some $q>1$ is sufficient for (iii). Finally, when $h_{n} \rightarrow 0$,

$$
\frac{1}{h_{n}} \operatorname{EK}\left(\frac{t-X_{1}}{h_{n}}\right) \rightarrow \mathrm{f}(\mathrm{x})
$$

is achieved with traditional techniques, and (complete convergence) consistency of the kernel density estimate in the $\mathrm{L}^{1}$-norm or the sup-norm is obtained from Theorem 2 .

The verification of (iv) depends on the particular Banach space. In particular, (iv) is implied by tightness and uniformly bounded $r^{\text {th }}$ moments $(r>1)$. It is easy to see that tightness and uniformly bounded $r^{\text {th }}$ moments $(r>1)$ are not necessary for Condition (iv) of Theorem 2 even when $E=R$.

The complete convergence for weighted sums of arrays of random elements will be obtained in Theorem 3. Only the parts of the proof which differ from the proof of Theorem 2 will be presented. First, let $\beta X$ denote the essential supremum of a nonnegative random variable $\mathrm{X}$.

THEOREM 3. Let $\left\{\mathrm{x}_{\mathrm{nk}}\right\}$ be an array of random elements in a separable Banach space E satisfying

(i) $\left\{\mathrm{x}_{\mathrm{nk}}: \mathrm{k} \geq 1\right\}$ are independent for each $\mathrm{n}$,

(ii) $\mathrm{EX}_{\mathrm{nk}}=0$ for $\mathrm{a} 11 \mathrm{n}$ and $\mathrm{k}$,

(iii) $\sup _{\mathrm{n}, \mathrm{k}} \beta|| \mathrm{x}_{\mathrm{nk}} \|=\Gamma<\infty$, and

(iv) given $\varepsilon>0$ there exists a compact set $K_{\varepsilon}$ such that

$$
\left.\underset{n}{\lim \sup } \sum_{\mathrm{k}=1}^{\infty}\left|\mathrm{a}_{\mathrm{nk}}\right| \mathrm{E}\left(|| \mathrm{x}_{\mathrm{nk}}|| \mathrm{I}_{\left[\mathrm{x}_{\mathrm{nk}}\right.} \notin \mathrm{K}_{\varepsilon}\right]\right)<\varepsilon
$$

Then

$$
\left\|\sum_{\mathrm{k}=1}^{\infty} \mathrm{a}_{\mathrm{nk}} \mathrm{x}_{\mathrm{nk}}\right\| \rightarrow 0 \text { completely. }
$$

OUTLINE OF PROOF. Steps $(2.6),(2.7)$, and (2.8) in the proof of Theorem 2 
remain the same. The random elements $\left\{|| z_{n k}\left\|-E|| z_{n k}\right\|\right\}$ have zero means and are bounded by $\Gamma$ with probability one. Hence, $\left\{|| z_{n k}\|-E\| z_{n k} \|\right\}$ are sub-Gaussian with parameters $\leq \Gamma 2^{1 / 2}$, and

$$
\left|\sum_{\mathrm{k}=1}^{\infty} \mathrm{a}_{\mathrm{nk}}\left(|| \mathrm{z}_{\mathrm{nk}}||-\mathrm{E}|| \mathrm{z}_{\mathrm{nk}}||\right)\right| \rightarrow 0
$$

completely by Theorem 2 of Chow [4]. Thus, the proof is completed by following the same steps of the proof of Theorem 2 .

Since $\sup _{k, n} \beta|| x_{n k}||<\infty$, tightness is sufficient for Condition (iii) of

Theorem 2. However, since $\lim \sup \sum_{k=1}^{\infty}\left|a_{n k}\right|=0$ is also sufficient, tightness is not necessary. Also, the more restrictive condition (iii) in Theorem 3 (than condition (iii) of Theorem 2) allows the use of more general weights $\left\{a_{n k}\right\}$.

\section{COMPLETE CONVERGENCE USING BASIS TECHNIQUES.}

In this section the complete convergence of weighted sums will be established for random elements in a Banach space which has a Schauder basis. Since the consistency of the kernel density estimates depend on a particular space which may have a basis, more general results can be obtained. The hypotheses will be shown to be more applicable when the Banach space has a Schauder basis. In particular, only coordinate-wise independence for some Schauder basis will be needed, and the weights $\left\{a_{n k}\right\}$ need only satisfy

$$
\sum_{n=1}^{\infty} \exp \left[-\alpha / A_{n}\right]<\infty
$$

for each $\alpha>0$ where $A_{n}=\sum_{\cdot k=1}^{\infty} a_{n k}^{2}$.

Let $\left\{b_{i}\right\}$ denote a Schauder basis for $E$ and let $\left\{f_{i}\right\}$ denote the corresponding coordinate functionals. For notation convenience let $U_{t}(x)=\sum_{i=1}^{t} f_{i}(x) b_{i}$ and $Q_{t}(x)=\sum_{i=t+1}^{\infty} f_{i}(x) b_{i}$ for each $t=1,2,3, \ldots$. It can be assumed without loss of generality that the basis is monotone (by renorming the space if necessary).

THEOREM 4. Let $\left\{\mathrm{x}_{\mathrm{nk}}\right\}$ be random elements in a Banach space which has a Schauder basis. If 
(i) for each $i,\left\{f_{i}\left(X_{n k}\right): k \geq 1\right\}$ are independent, sub-Gaussian random variables for each n-with uniformly bounded parameters $\tau\left(f_{i}\left(x_{n k}\right)\right)$,

(ii) $\quad \mathrm{EX}_{\mathrm{nk}}=0$ for each $\mathrm{n}$ and $\mathrm{k}$, and

(iii) $\quad \lim _{t \rightarrow \infty} \sup _{n} \beta\left\|Q_{t}\left(\sum_{k=1}^{\infty} a_{n k} x_{n k}\right)\right\|=0$,

then

$$
\left\|\sum_{k=1}^{\infty} a_{n k} x_{n k}\right\| \rightarrow 0 \quad \text { completely. }
$$

PROOF. Given $\varepsilon>0$ pick $t$ so that

$$
\sup _{\mathrm{n}}\left\|\sum_{\mathrm{k}=1}^{\infty} a_{\mathrm{nk}} Q_{t}\left(X_{n k}\right)\right\|<\frac{\varepsilon}{2}
$$

with prohability one. Thus, for each $n$

$$
\begin{aligned}
& P\left[\left\|\left.\right|_{k=1} ^{\infty} a_{n k} x_{n k}\right\|>\varepsilon\right] \\
& \leq P\left[\left\|U_{t}\left(\sum_{k=1}^{\infty} a_{n k} x_{n k}\right)\right\|>\frac{\varepsilon}{2}\right] \\
& \quad \leq \sum_{i=1}^{t} P\left[\left|\sum_{k=1}^{\infty} a_{n k} f_{i}\left(x_{n k}\right)\right|>\frac{\varepsilon}{2 t}\right] .
\end{aligned}
$$

ence, from (i), (ii), (3.1), and (3.3)

$$
\sum_{n=1}^{\infty} P\left[|| \sum_{k=1}^{\infty} a_{n k} x_{n k} \|>\varepsilon\right]<\infty .
$$

Theorem 4 allows for more general weights since $\sum_{k=1}^{\infty}\left|a_{n k}\right|=b_{n}$ need not be bounded. Note that in this case $\sum_{k=1}^{\infty} a_{n k} x_{n k}$ is assumed to be a.s. convergent. Condition (i) is easily satisfied in general. For example, if $P\left[X_{n k} \in K\right]=1$ for each $n$ and $k$ where $k$ is a compact set, then $\left\{f_{i}\left(x_{n k}\right)\right\}$ are sub-Gaussian with parameter $\tau\left(f_{i}\left(x_{n k}\right) \leq 2^{1 / 2}\left\|f_{i}\right\|\left(\sup _{x \in K}\|x\|\right)\right.$. Also, by Lemma 1.3 .3 of Taylor [3] $\lim _{t \rightarrow \infty} \sup _{n, k} \beta\left\|Q\left(X_{n k}\right)\right\|=0$ when $P\left[X_{n k} \in K\right]=1$. Thus, Condition (iii) also holds in this case when $\sum_{\cdot k=1}^{\infty}\left|a_{n k}\right| \leq 1$. Finally, as an aside, the conclusion of Theorem 4 holds almost surely (rather than completely) if the uniform almost sure convergence of Condition (iii) is replaced by simply almost sure convergence.

Coordinate-wise sub-Gaussian random elements are also appealing for applications since coordinate-wise independence suffices in this setting. In general, condition 
(iii) is the troublesome requirement which must be satisfied. The following lemma (a modification of Lemma 8.1 from Zaanen [6])allows (iii) to be replaced by a more restrictive condition but one which may be easier to verify in applications.

LEMMA. Let $\left\{\mathrm{Y}_{\mathrm{tn}}: \mathrm{t}, \mathrm{n}=1,2, \ldots\right\}$ be real-valued random variables satisfying

(i) $\mathrm{Y}_{\mathrm{tn}} \geq 0$ with probability one for all $\mathrm{t}$ and $\mathrm{n}$,

(ii) for fixed $n, Y_{(t+1) n} \leq Y_{t n}$ with probability one for each $t=1,2, \ldots$, and

(iii) $\mathrm{Y}_{\mathrm{tn}} \leq \mathrm{Y}$ with probability one for each $\mathrm{t}$ and $\mathrm{n}$ where $\mathrm{Y}$ is a random variable such that $E Y<\infty$.

Then, $\lim _{t \rightarrow \infty} \sup \mathrm{EY}_{\mathrm{tn}}=0$ implies that $\lim _{t \rightarrow \infty} \mathrm{E}\left[\sup \mathrm{Y}_{\mathrm{tn}}\right]=0$.

THEOREM 5. Let $\left\{\mathrm{X}_{\mathrm{nk}}\right\}$ be random elements in a Banach space which has a monotone basis. If

(i) \& (ii) (same as Condition (i) and (ii) of Theorem 4),

(iii) $\lim _{t \rightarrow \infty} \sup _{n} E\left[\left\|Q_{t}\left(\sum_{k=1}^{\infty} a_{n k} x_{n k}\right)\right\|\right]=0$, and

(iv) $\quad E\left[\sup _{n}\left\|\sum_{k=1}^{\infty} a_{n k} x_{n k}\right\|\right]<\infty$.

Then,

$$
\left\|\sum_{\mathrm{k}=1}^{\infty} \mathrm{a}_{\mathrm{nk}} \mathrm{x}_{\mathrm{nk}}\right\| \rightarrow 0 \text { a.s. }
$$

PROOF. Condition (iv) implies that $\sum_{k=1}^{\infty}{ }^{a}{ }_{n k} x_{n k}$ is defined with probability one. With probability one, $\sup _{n}\left\|\sum_{k=1}^{\infty} a_{n k} x_{n k}\right\|$ can be identified as $\mathrm{Y}$ in the preceding lemma. Since $E$ has a monotone basis, $Y_{t n}=\left\|Q_{t}\left(\eta_{k=1}^{\infty} a_{n k} X_{n k}\right)\right\|$ satisfies (ii) of the lemma. Next let $\mathrm{Y}_{t}$ be identified as $\sup _{n}\left\|\mathrm{Q}_{\mathrm{t}}\left(\sum_{\mathrm{k}=1}^{a} a_{\mathrm{nk}} \mathrm{x}_{\mathrm{nk}}\right)\right\|$ with probability one. Thus, (iii) implies that $\underset{t \rightarrow \infty}{\lim } \operatorname{E}\left[\sup _{n}\left\|Q_{t}\left(\sum_{k=1}^{\infty} a_{n k} x_{n k}\right)\right\|\right]=$ $\lim _{t \rightarrow \infty} E Y_{t}=0$. Hence, $\underset{t \rightarrow \infty}{\lim } Y_{t}=0$ in probability, and $\underset{m \rightarrow \infty}{\lim } Y_{t_{m}}=0$ with probability one 
for some subsequence $\left\{t_{m}\right\}$. Since $0 \leq Y_{t}=\sup _{n}\left\|Q_{t}\left(\sum_{k=1}^{\infty} a_{n k} X_{n k}\right)\right\|$ is nonincreasing with probability one, it follows that $\underset{t \rightarrow \infty}{\lim } \mathrm{Y}_{t}=$

$\lim _{t \rightarrow \infty} \sup _{n}\left\|Q_{t}\left(\sum_{k=1}^{\infty} a_{n k} X_{n k}\right)\right\|=0$ with probability one. Theorem 5 now follows from a modification of Theurem 4 .

The comparisons of general Banach space results with the results of this section will be completed by showing that condition (iv) of Theorem 3 implies condition (ii) of Theorem 5. Assume that $\sum_{k=1}^{\infty}\left|a_{n k}\right| \leq 1$ for each $n$ and that for $\varepsilon>0$ a compact, convex, symmetric set $K$ can be chosen so that $0 \epsilon \mathrm{K}$ and

$$
\left.\sup _{n} \sum_{k=1}^{\infty}\left|a_{n k}\right| E\left[|| x_{n k}|| I_{X_{n k}} \notin k\right]\right]<\frac{\varepsilon}{2} .
$$

By Lemma 1.3 .3 of Taylor [3], choose $t_{0}$ so that for all $t \geq t_{0}$

$\sup _{x \in K}\left\|Q_{t}(x)\right\|<\frac{\varepsilon}{2}$. Then for $t \geq t_{0}$

$$
\left\|Q_{t}\left(\sum_{k=1}^{\infty} a_{n k} X_{n k} I_{\left[X_{n k} \in K\right]}\right)\right\|<\frac{\varepsilon}{2}
$$

since $\sum_{k=1}^{\infty}\left|a_{n k}\right| \leq 1$ for each $n$. Then for $t \geq t_{0}$

$$
\begin{array}{rl}
\sup _{n} & E\left[\left\|Q_{t}\left(\sum_{k=1}^{\infty} a_{n k} X_{n k}\right)\right\|\right] \\
& \leq \sup _{n} E\left[\left\|Q_{t}\left(\sum_{k=1}^{\infty} a_{n k} X_{n k} I_{\left[X_{n k} \in K\right]}\right)\right\|\right] \\
& +\sup _{n} \sum_{k=1}^{\infty}\left|a_{n k}\right| E\left[|| Q_{t}\left(x_{n k}\right)|| I_{\left[X_{n k}\right.} k K\right] \\
& <\frac{\varepsilon}{2}+\frac{\varepsilon}{2}=\varepsilon
\end{array}
$$

from (3.4) and (3.5) since ||$Q_{t}(x)\|\leq\| x \|$ for all $t$. Thus, the compact uniform integrability of (3.4) (and hence tightness with uniformly bounded $\mathrm{p}^{\text {th }}(\mathrm{p}>1)$ moments) is sufficient for Condition (iii) of Theorem 5. Identical distributions and moments conditions will suffice for Condition (iii). However, while it is often true (in applications) that $\left\{\mathrm{x}_{\mathrm{nk}}: \mathrm{k} \geq 1\right\}$ are independent and identically distributed for each $\mathrm{n}$, identical distributions seldom hold for the array $\left\{\mathrm{x}_{\mathrm{nk}}\right\}$. 


\section{ACKNOWLEDGEMENTS.}

The research was supported in part by the Air Force Office of Scientific Research under Contract Number F49620-79-C-140 and Grant Number AFOSR-81-0166. Helpful editorial changes and suggestions are also gratefully acknowledged.

\section{REFERENCES}

[1] WEI, D. and TAYLOR, R. L. Convergence of weighted sums of tight random elements, J. Multivariate Anal., 8 , (1978), 282-294.

[2] HOWELL, J. O. \& TAYLOR, R. L. Marcinkiewicz-Zygmund weak laws of large numbers for unconditional random elements in Banach spaces. Probability in Banach Spaces III LECTURES NOTES IN MATHEMATICS Vo1 860, (1981), Springer-Verlag, 219-230.

[3] TAYLOR, R. L. Stochastic Convergence of Weighted Sums of Random Elements in Linear Spaces, Springer-Verlag Lecture Notes, Vol. 672, (1978).

[4] CHOW, Y. S. Some convergence theorems for independent random variables, Annals Math. Statist., 37 (1966), 1482-1493.

[5] WOYCZYNSKI, W. On Marcinkiewicz-Zygmund laws of large numbers in Banach spaces and related rates of convergence, Prob. and Math. Statist., 1, (1980), 117-132.

[6] ZAANEN, A. C. The Radon-Nikodym theorem - II, Indag. Math., 23, (1961), 171-187. 


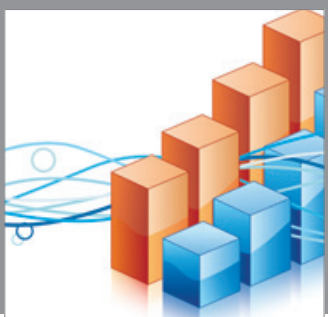

Advances in

Operations Research

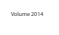

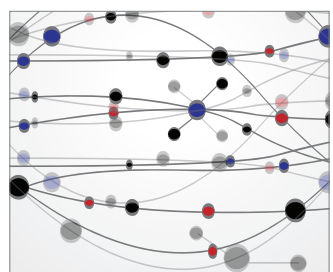

\section{The Scientific} World Journal
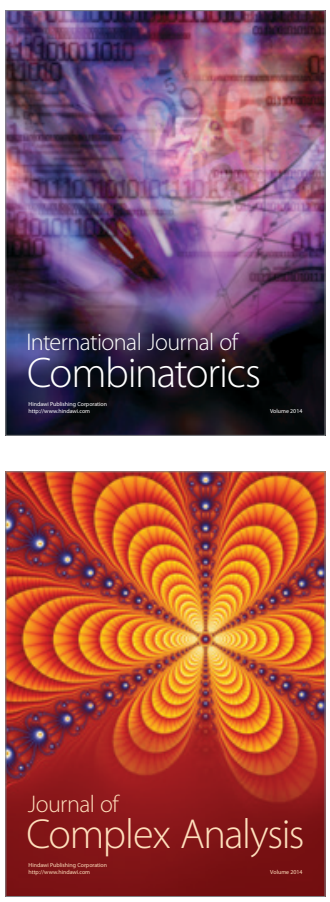

International Journal of

Mathematics and

Mathematical

Sciences
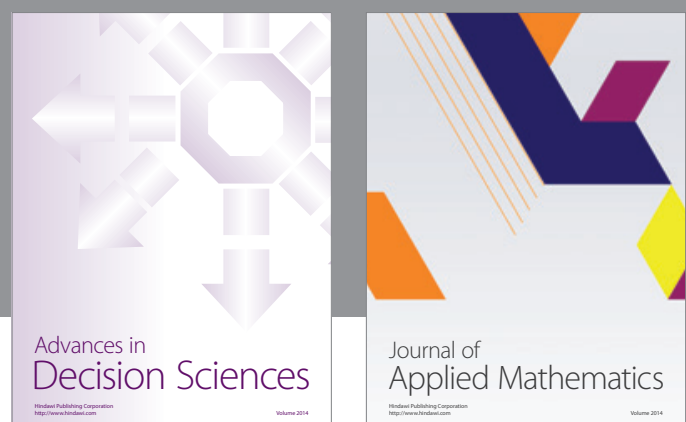

Journal of

Applied Mathematics
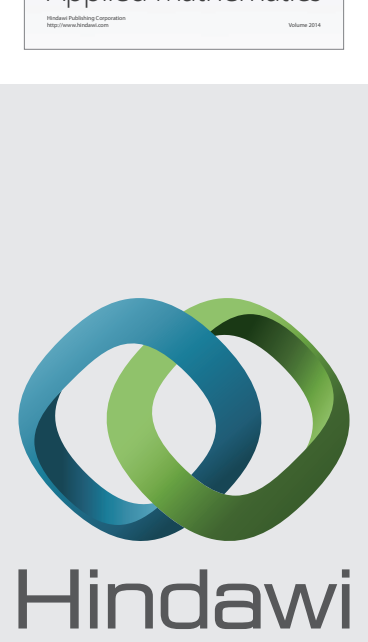

Submit your manuscripts at http://www.hindawi.com
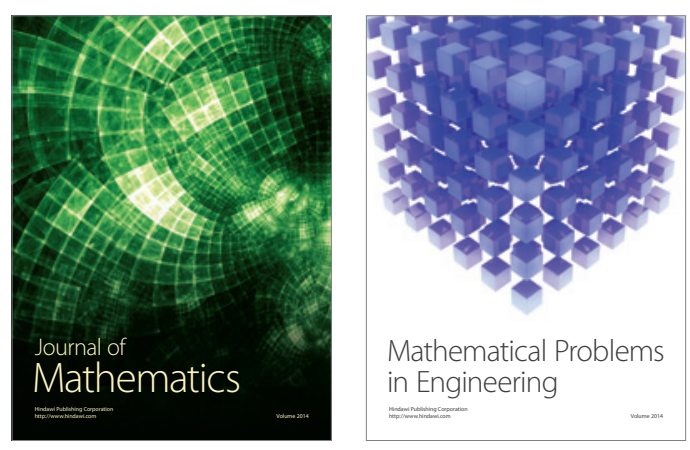

Mathematical Problems in Engineering
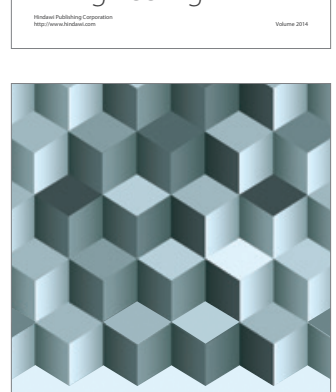

Journal of

Function Spaces
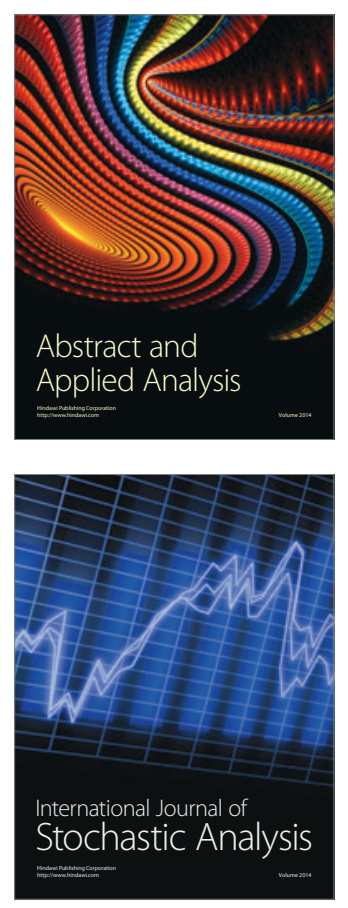

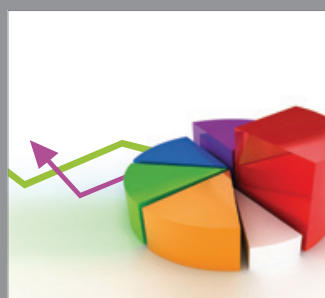

ournal of

Probability and Statistics

Promensencen
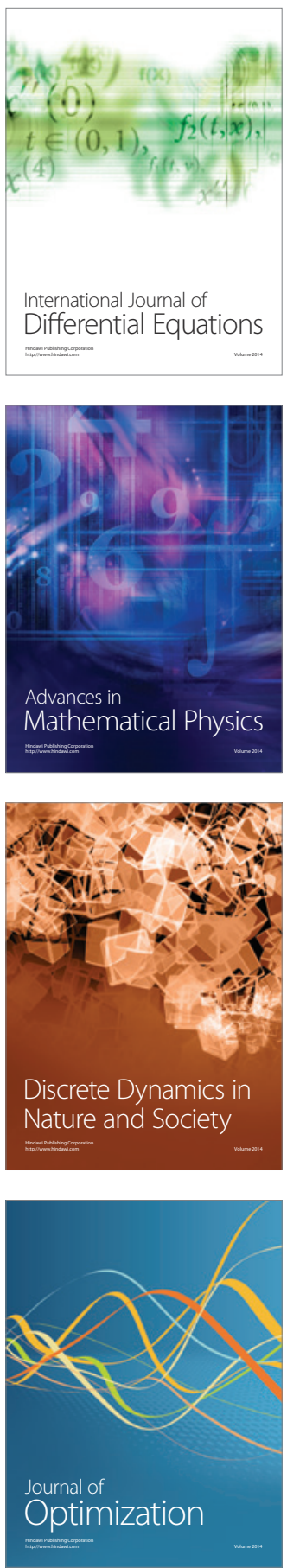